\title{
Endocytic Accessory Factors and Regulation of Clathrin-Mediated Endocytosis
}

\author{
Christien J. Merrifield ${ }^{1}$ and Marko Kaksonen ${ }^{2}$ \\ ${ }^{1}$ Laboratoire d'Enzymologie et Biochimie Structurales, Centre National de la Recherche Scientifique \\ UPR3082, 91198 Gif-sur-Yvette, France \\ ${ }^{2}$ Cell Biology and Biophysics Unit, European Molecular Biology Laboratory, 69117 Heidelberg, \\ Germany \\ Correspondence: kaksonen@embl.de; christien.merrifield@lebs.cnrs-gif.fr
}

\begin{abstract}
Up to 60 different proteins are recruited to the site of clathrin-mediated endocytosis in an ordered sequence. These accessory proteins have roles during all the different stages of clathrin-mediated endocytosis. First, they participate in the initiation of the endocytic event, thereby determining when and where endocytic vesicles are made; later they are involved in the maturation of the clathrin coat, recruitment of specific cargo molecules, bending of the membrane, and finally in scission and uncoating of the nascent vesicle. In addition, many of the accessory components are involved in regulating and coupling the actin cytoskeleton to the endocytic membrane. We will discuss the different accessory components and their various roles. Most of the data comes from studies performed with cultured mammalian cells or yeast cells. The process of endocytosis is well conserved between these different organisms, but there are also many interesting differences that may shed light on the mechanistic principles of endocytosis.
\end{abstract}

$\mathrm{R}^{\mathrm{e}}$ eceptor-mediated endocytosis is the process by which eukaryotic cells concentrate and internalize cell surface receptors from the plasma membrane into small $(\sim 50 \mathrm{~nm}-$ $\sim 100 \mathrm{~nm}$ diameter) membrane vesicles (Chen et al. 2011; McMahon and Boucrot 2011; Weinberg and Drubin 2012). This mechanism has been studied extensively in mammalian tissue culture cells and in yeast, and despite the evolutionary distance between yeast and mammalian cells the mechanism of receptor-mediated endocytosis in the respective cell types show remarkable similarities. Indeed many of the $\sim 60$ endocytic accessory proteins (EAPs) found in yeast have homologs in mammalian cells, al- though both cell types also have unique EAPs (McMahon and Boucrot 2011; Weinberg and Drubin 2012).

In the following, we briefly describe known yeast and mammalian EAPs (Table 1). We then focus on recent efforts toward integrated models describing how EAPs are spatially and temporally organized to form endocytic vesicles. Many of these more recent studies have exploited advances in fluorescence microscopy and the use of fluorescent proteins (FPs) in live-cell imaging. We do not discuss the relationship between receptor-mediated endocytosis and signaling, which has been covered by recent reviews (Sigismund et al. 2012; see also Bökel

Editors: Sandra L. Schmid, Alexander Sorkin, and Marino Zerial

Additional Perspectives on Endocytosis available at www.cshperspectives.org

Copyright (C) 2014 Cold Spring Harbor Laboratory Press; all rights reserved; doi: 10.1101/cshperspect.a016733

Cite this article as Cold Spring Harb Perspect Biol 2014;6:a016733 
C.J. Merrifield and M. Kaksonen

Table 1. Key endocytic proteins in mammals and in yeast

\begin{tabular}{|c|c|c|c|}
\hline & Mammals & Yeast & Function \\
\hline \multirow[t]{11}{*}{ Coat proteins } & Clathrin & Chc1, Clc1 & Coat protein \\
\hline & AP-2 (4 subunits) & AP-2 (4 subunits) & Adaptor protein \\
\hline & Epsin & Ent $1 / 2$ & Adaptor protein \\
\hline & AP180 & Yap $1801 / 2$ & Adaptor protein \\
\hline & CALM & - & Adaptor protein \\
\hline & NECAP & - & Adaptor protein \\
\hline & $\mathrm{FCHo} 1 / 2$ & Syp1 & Adaptor protein \\
\hline & Eps15 & Ede1 & Scaffold protein \\
\hline & Intersectin & Pan1 & Scaffold protein \\
\hline & - & Sla1 & Scaffold protein \\
\hline & - & End3 & Scaffold protein \\
\hline \multirow[t]{3}{*}{ N-BAR proteins } & Amphiphysin & Rvs161/167 & Membrane curvature sensor/generator \\
\hline & Endophilin & - & Membrane curvature sensor/generator \\
\hline & BIN1 & - & Membrane curvature sensor/generator \\
\hline Dynamin & Dynamin $1 / 2$ & Vps1 & Mechanoenzyme, GTPase \\
\hline \multirow[t]{6}{*}{ Actin cytoskeleton } & Actin & Act1 & Actin monomer \\
\hline & Arp2/3 complex & Arp2/3 complex & Actin filament nucleator \\
\hline & ABP1 & Abp1 & Actin-binding protein \\
\hline & Cortactin & - & Actin-binding protein \\
\hline & Coronin & Crn1 & Actin-binding protein \\
\hline & Cofilin & Cof1 & Actin depolymerizing protein \\
\hline \multirow[t]{8}{*}{ Actin regulators } & Myosin 1E & Myo3/5 & Actin motor \\
\hline & Myosin 6 & & Actin motor \\
\hline & Hip1R, Hip1 & Sla2 & Actin-membrane coupler \\
\hline & Syndapin & Bzz1 & BAR domain protein \\
\hline & N-WASP & Las17 & Regulator of actin nucleation \\
\hline & WIP/WIRE & Vrp1 & Regulator of actin nucleation \\
\hline & SNX9 & - & Regulator of actin nucleation \\
\hline & - & Bbcl & Regulator of actin nucleation \\
\hline \multirow[t]{4}{*}{ Other regulators } & AAK1 & Ark1/Prk1 & Protein kinase \\
\hline & Auxilin, GAK & - & Uncoating factor \\
\hline & Synaptojanin & Sj12 & Lipid phosphatase \\
\hline & OCRL1 & - & Lipid phosphatase \\
\hline
\end{tabular}

The proteins are grouped into functional categories and the homologous proteins are listed on the same line.

and Brand 2013; Cosker and Segal 2014; Di Fiore and von Zastrow 2014).

\section{A MODULAR DESIGN FOR YEAST AND MAMMALIAN ENDOCYTOSIS}

In some respects, the endocytic machinery resembles a 3D jigsaw puzzle in which many of the pieces can interact with more than one of the other pieces and the size, composition, and shape of the jigsaw puzzle changes with time. Merely knowing the pieces does not tell you how the machine is organized.
Advances in live-cell fluorescence imaging techniques and the introduction of fluorescent proteins enabled the dynamics of endocytic accessory proteins to be monitored in live cells (Kirchhausen 2009). In yeast EAPs are recruited to, and dismissed from, sites of endocytosis in a stereotyped sequence relative to the inward movements of endocytic buds (Kaksonen et al. 2005; Carroll et al. 2012). Based on the spatiotemporal patterns of recruitment of endocytic accessory factors to sites of endocytosis, five distinct functional modules of proteins were identified: (1) an early module (Ede1, Syp1), (2) a 
Endocytic Accessory Factors and Regulation of CME

coat module (clathrin, End3, Sla1, Pan1), (3) a WASP/Myo module (Bbc1, Myo5, Las17), (4) an amphiphysin module (Rvs161, Rvs167), and (5) an actin module (Sac6, Cap1, Cap2, Abp1, actin, Arp3) (Fig. 1). The precise role(s) of the different modules in endocytic bud formation are described in detail later in this review.

Unlike yeast cells, until recently it was not possible to replace endogenous genes in mammalian cells with their FP tagged cognates (but see Doyon et al. 2011). Instead low overexpression levels of endocytic accessory proteins were used, although this naturally raised concerns over potential artifacts caused by protein overexpression (Doyon et al. 2011; although see Aguet et al. 2013). Nonetheless, the recruitment of FP tagged endocytic accessory proteins was measured relative to single productive scission events in one cell type (mouse fibroblasts) with a temporal resolution of $\sim 2 \mathrm{sec}$ (Merrifield et al. 2005; Taylor et al. 2011, 2012). Using cluster analysis to group protein-FP recruitment profiles, it was found that there were approximately seven natural groups of accessory protein recruitment kinetics corresponding to approximately four different protein modules: (1) the coat module, divided into (i) a clathrin submodule (epsin2, clathrin light chain, and NECAP), and (ii) an adaptor/F-BAR submodule (FCHo1/2, Eps15, AP2); (2) the NBAR domain module (endophilin2, amphiphysin2, and BIN1); (3) the actin module including (i) actin polymerization submodule (Abp1, cortactin, and Arp3), and (ii) actin depolymerization/ suppression (cofilin, coronin1B, and SNX9); (4) the dynamin/myosin/N-WASP module (dynamin1/2, myosin/N-WASP, Eps8, Hip1R, myosin6, and syndapin2); (5) the GAK postscission module (GAK, ACK1, and OCRL1); (6) the Rab5a module (Rab5a and APPL1); and (7) the Fbp17/CIP4 module (Table 1) (Taylor et al. 2011).

The structural homologies between many mammalian and equivalent yeast EAPs are striking and the assembly dynamics of the EAPs appear overall rather similar. However, there are many intriguing differences in the spatiotemporal organization of the mammalian and yeast endocytic machinery. For instance, in mammalian cells clathrin is thought to be an integral part of the membrane-curvature generating mechanism required for clathrin-coated pit (CCP) in-

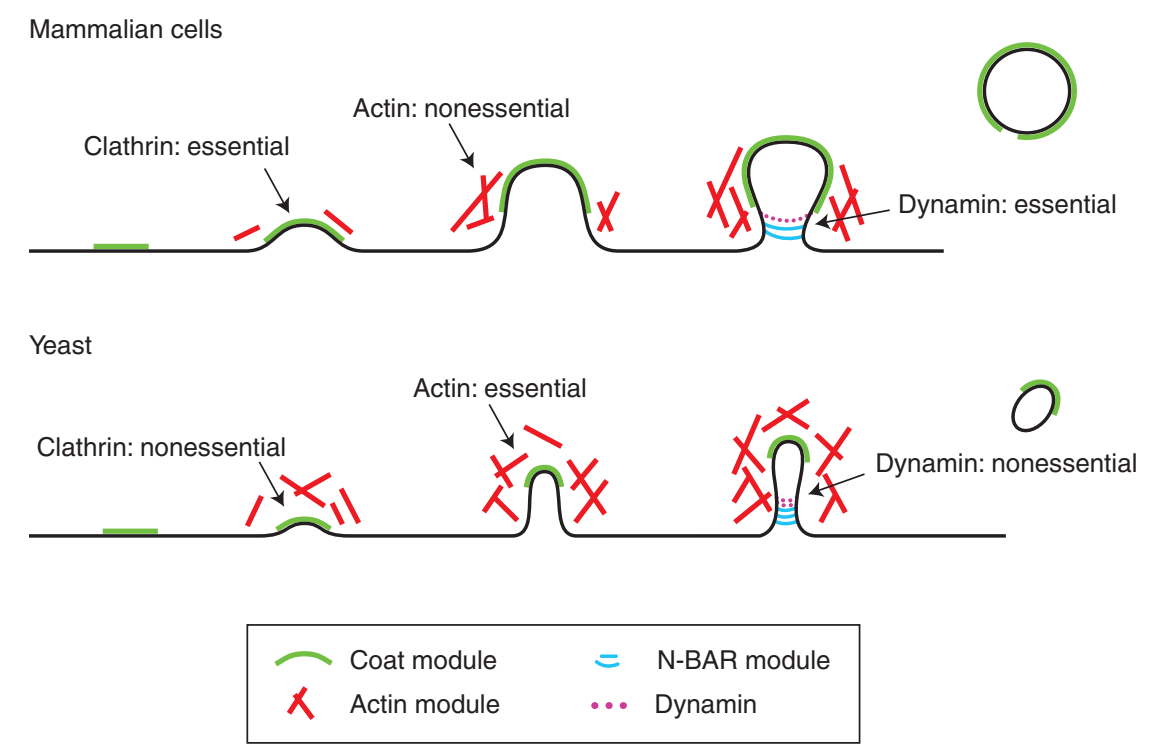

Figure 1. Clathrin-mediated endocytosis in mammalian and yeast cells. The basic sequence of events is similar but the requirements for clathrin, actin, and dynamin differ between these organisms. The shape of the endocytic intermediates is approximately spherical in mammals, but tubular in yeast. 
vagination (Dannhauser and Ungewickell 2012; Kirchhausen 2012). In contrast, in yeast cells clathrin is not obligatory for receptor-mediated endocytosis (Payne et al. 1988). Rather, clathrin is recruited to the site of bud initiation when the membrane is still flat and membrane curvature is tightly correlated with actin polymerization (Kukulski et al. 2012). Finally, there are apparently striking differences in the mechanism of membrane scission in mammalian and yeast cells. In mammalian cells, membrane scission absolutely requires the large GTPase dynamin (Ferguson and De Camilli 2012) whereas in yeast cells actin, rather than an equivalent GTPase is important for scission (Kishimoto et al. 2011). The mechanistic similarities and differences between mammalian and yeast endocytosis are summarized in Figure 1.

Why are there differences in the organization of mammalian and yeast endocytosis? This may be attributable to physical constraints placed on the endocytic machinery in these two very different types of cells. For instance, it has been proposed that actin plays an obligatory role in walled yeast endocytosis because high membrane tension resulting from turgor pressure elicits more work to invaginate the membrane as compared with mammalian cells (Meckel et al. 2004; Aghamohammadzadeh and Ayscough 2009; Boulant et al. 2011). Indeed, there is evidence that membrane tension may modulate the requirement for actin in mammalian $\mathrm{CME}$ and yeast protoplasts wherein regimes of high membrane tension, generated by hyperosmotic challenge or physical stretching, invoke a requirement for actin (Aghamohammadzadeh and Ayscough 2009; Boulant et al. 2011). The differences in turgor pressure may also explain the differences in the shapes of endocytic membrane invaginations, which are roughly spherical in mammalian cells but tubular in yeast (Idrissi et al. 2012; Kukulski et al. 2012).

\section{EAPs AND ENDOCYTIC SITE NUCLEATION: WHENCE THE PITS?}

A simple question in the biology of CME is how CCPs start (Ehrlich et al. 2004; Antonescu et al. 2010; Henne et al. 2010; Nunez et al. 2011; Co- cucci et al. 2012; Merrifield 2012; Umasankar et al. 2012). Much of the spatial and temporal regulation of endocytosis is probably conducted by regulating the initiation of CCP assembly. Many EAPs, including adaptor proteins, contain basic motifs adapted to bind negatively charged phosphatidylserine (PS) and phosphatidylinositol-4,5-bisphosphate (PIP2) concentrated in the inner leaflet of the plasma membrane (Antonescu et al. 2010). One might thus speculate a priori that the association of negatively charged phospholipids, adaptor proteins, receptor cargo, and coat proteins at the inner leaflet of the plasma membrane would be the first event in coated bud formation, and this has indeed been the central tenet in investigations of CCP nucleation.

First, the evidence for a role for negatively charged phospholipids in bud nucleation in both yeast and mammalian cells is well-established (Jost et al. 1998; Abe et al. 2008; Zoncu et al. 2009; Antonescu et al. 2010; Sun and Drubin 2012). In mammalian cells, depletion of plasma membrane PIP2 by pharmacological challenge with the $\mathrm{Ca}^{2+}$ ionophore ionomycin or by the engineered translocation of a PI5phosphatase to the plasma membrane triggered a dramatic loss of CCPs and abolished CCP initiation events as measured using clathrin light chain-fluorescent protein (Clc-FP) or adaptor protein complex-2-fluorescent protein (AP2FP) probes (Zoncu et al. 2009; although see Abe et al. 2008). By probing the cytoplasmic face of the plasma membrane with a GST-PH probe, gold labeling, and observation by EM, it was shown that PIP2 was significantly more concentrated at the rim of CCPs than on the surrounding, flat membrane or the apex of the CCP invagination (Fujita et al. 2009). It will be interesting to find, using alternative PI specific probes, whether other PIs are concentrated at CCPs with a similarly heterogeneous distribution.

Where does the PIP2 concentrated at CCPs come from? It seems unlikely that localized PIP2 production contributes to CCP initiation or maturation because no PIP2-kinases have been localized to CCPs (Sun et al. 2007; Antonescu et al. 2010). In contrast a variety of $\operatorname{PIP}_{2}\left(5^{\prime}\right)$ phosphatases (OCRL, Sjn1, and Sjn2) do local- 
ize to CCPs and play important roles in CCP maturation and budding (Perera et al. 2006; Mao et al. 2009; Nakatsu et al. 2010, 2012; Taylor et al. 2011). A model has therefore emerged in which PIP2 diffusing in the plasma membrane is sequestered at the CCP nucleation site through interaction with a variety of PIP2-binding EAPs and wherein PIP2 turnover plays a key role in CCP maturation and scission (Antonescu et al. 2010). In a very recent development it was discovered that $\mathrm{PI}(3,4) \mathrm{P}_{2}$ plays an essential role in the later stages of CCP invagination and transition from invaginated to $\Omega$ shaped CCPs (Posor et al. 2013). The $\operatorname{PIP}(3,4) \mathrm{P}_{2}$ concentrated at CCPs is produced locally from $\mathrm{PI}(4,5) \mathrm{P}_{2}$ by the concerted action of PI5 phosphatases and $\mathrm{PI} 3 \mathrm{~K} \mathrm{C} 2 \alpha$ and is required for recruitment of the BAR domain protein sorting nexin 9 (SNX9) (Posor et al. 2013). It should be noted that PI4P may also contribute to the recruitment of EAPs because $\mathrm{PI}(4,5) \mathrm{P}_{2}$ and PI4P have some degree of overlap in defining the electrostatic properties of the inner leaflet of the plasma membrane (Hammond et al. 2012). The phosphoinositide PI4P has been associated with clathrin-coated vesicle (CCV) uncoating, so precisely how PIP2, $\mathrm{PI}(3,4) \mathrm{P}_{2}$, and PI4P are coordinated at CCPs remains an intriguing area of investigation (Nakatsu et al. 2012; Posor et al. 2013). In yeast, negatively charged phospholipids play a similarly important role in CCP nucleation although here PS, rather than PIP2, is essential for endocytic bud site selection and initiation, whereas PIP2 is required for efficient invagination (Sun and Drubin 2012).

Second, the role of adaptor proteins in bud nucleation in both mammalian and yeast cells is generally clear (Motley et al. 2003; Antonescu et al. 2010; Cocucci et al. 2012), although some areas of controversy remain. In mammalian cells a plethora of adaptor proteins have been described (Fig. 1), although the AP2 complex is the best studied and most important. Knockdown of AP2, the main adaptor protein complex in mammalian cells, led to a dramatic loss of CCPs, abrogation of TfR (Motley et al. 2003) and EGFR internalization (Goh et al. 2010), and a correlate reduction in CCP nucleation events (Motley et al. 2003). However, a small popula- tion $(\sim 1 / 12$ th the original) of CCPs nucleated in the apparent absence of AP2 and remained competent, for epidermal growth factor receptor (EGFR) internalization (Motley et al. 2003). It has been suggested that these CCPs retain small amounts of the residual AP2 complexes (Boucrot et al. 2010) which may be enough to nucleate CCP formation. Thus, whereas there remains some controversy, it seems likely that AP2 complexes play an essential role in the nucleation of most CCPs and AP2 may serve as a paradigm for the role played by adaptors in CCP nucleation. The distribution of PIP2 in the inner leaflet of the mammalian plasma membrane is uniform in nonexcitable cells (Antonescu et al. 2010) and AP2 has a relatively low affinity for PIP2, leading to the suggestion that AP2 "samples" the plasma membrane (Cocucci et al. 2012). On binding PIP2 the AP2 complex undergoes a dramatic structural rearrangement and unfurls to reveal cargo and clathrin-binding motifs (Jackson et al. 2010). If the AP2 complex then successfully binds receptor cargo and clathrin further PIP2-binding motifs are revealed, association with the membrane strengthens and nucleation of a nascent CCP begins (Jackson et al. 2010). In this model, the AP2 complex therefore acts as a coincidence detector directly linking PIP2, receptor cargo, and clathrin. Similarly, in yeast Edelp (Eps15 homolog), clathrin, the AP2 complex, and Syplp (FCHo homolog) are recruited early to nascent endocytic sites (Burston et al. 2009; Carroll et al. 2009; Reider et al. 2009). Deleting genes for Syp1, AP2 complex, or Yap1801/2 does not cause defects in endocytic dynamics but these proteins are important for the internalization of certain cargo (Burston et al. 2009; Carroll et al. 2009; Reider et al. 2009). The yeast scaffold protein Edelp (Eps15 homolog) was found necessary for recruitment of the majority of earlyarriving proteins barring the adaptor protein Yap1802p (Carroll et al. 2012).

Third, the (perhaps self-evident) role of clathrin in metazoan CME has been clearly shown and knockdown of clathrin heavy chain using siRNA led to a loss of coated pits and a concomitant decrease in both TfR and EGFR endocytosis (Motley et al. 2003; Huang et al. 
2004). However, controlled elimination of clathrin heavy chain expression in chicken DT40 lymphocytes abrogated, but surprisingly did not entirely abolish, TfR recycling although the majority of cells died via apoptosis (Wettey and Jackson 2006). Unlike metazoan in yeast cells it was shown that clathrin is important, but not obligatory, for receptor-mediated endocytosis (Payne et al. 1988; Tan et al. 1993; Carroll et al. 2012). In CLC-1 knockout yeast, the later arriving proteins Sla2p (Hip1R homolog) and Ent1/2p (epsin homologs) were found to have longer lifetimes indicating that clathrin facilitates the transition from intermediate to late coat stages (Carroll et al. 2012).

Finally, the role of receptor cargo in CCP nucleation in mammalian cells has been clearly shown. Overexpression of the TfR did not increase the nucleation rate of CCPs but did increase the probability of CCPs successfully maturing (Loerke et al. 2009). However, an elegant experiment by Schmid and coworkers showed that local clustering of biotinylated TfR into tetramers using fluorescent streptavidin did promote CCP nucleation, suggesting that localized concentration of signaling motifs promoted CCP nucleation by recruiting adaptor proteins (Liu et al. 2010). The picture in yeast cells is less clearly defined and, unlike mammalian cells, the arrival of the receptor cargo, $\alpha-$ factor apparently occurred after the initiation of bud nucleation (Toshima et al. 2006).

Although the studies described have shown that anionic phospholipids, adaptor proteins, cargo, and coat proteins are necessary for bud initiation, the precise mechanism of the very earliest steps in CCP nucleation has remained obscure.

In mammalian cells, an elegant series of single molecule TIR-FM imaging experiments described the earliest $5 \mathrm{sec}$ in the life of CCPs (Cocucci et al. 2012). Importantly, the stoichiometry of adaptors, clathrin, and receptor cargo could be inferred through careful quantification of the stepwise increases in FP tagged EAPs at nascent CCPs. It was shown that the nucleation of discrete, punctate CCPs occurred stochastically by minimal association of PIP2, 2AP2 molecule, and 1 clathrin triskelion (Cocucci et al. 2012), similar to predictions presented in earlier studies.

An alternative hypothesis to the "AP2-centric" model of CCP nucleation is that CCPs are nucleated by a specialized "nucleation complex" consisting of receptor cargo, PIP2, the adaptors AP2 and Eps15, and the F-BAR protein FCHo (Henne et al. 2010). This hypothesis in particular addressed the earliest stages of coat invagination and it was proposed that FCHo, a curvature-inducing F-BAR protein (Henne et al. 2007; Shimada et al. 2007) implicated in clathrin-mediated endocytosis (Sakaushi et al. 2007), might induce a "pimple" in the plasma membrane and so initiate the formation of a curved clathrin coat (Henne et al. 2010). However, subsequent experiments in zebrafish embryos and mammalian cultured cells suggested FCHo is nonessential for general CME (Shimada et al. 2007; Cocucci et al. 2012) and is rather an adaptor for BMP receptor (Umasankar et al. 2012) and/or facilitates CCP invagination (Gaidarov et al. 1999; Shimada et al. 2007; Cocucci et al. 2012).

In yeast cells a different strategy was used to capture the earliest events in bud formation. Correlative fluorescence microscopy and electron tomography (ET) was used to link the ultrastructure of endocytic buds to the relative time point in endocytic bud progression $(\mathrm{Ku}-$ kulski et al. 2012). Using Abp1-mRFP as a constant marker, cell lines coexpressing Ede1-GFP (Eps15), Sla1-GFP (Hip1R), and Rvs167-GFP (amphiphysin) were generated. By measuring the relative fluorescence of the Abp1-mRFP and corresponding GFP signal at randomly selected endocytic patches from the three cell lines, nine contiguous time windows were defined and mapped to the corresponding bud topology. When the early module or coat module was present in the absence of actin only flat membrane was found. After the initiation of actin polymerization, measured by Abp 1-mRFP fluorescence, $>99 \%$ of endocytic sites showed either an invagination or vesicle. These results suggest that curvature is not initiated at the point of coat recruitment but rather at the moment when actin polymerization begins at the endocytic site. Surprisingly, clathrin was found to be present on 
the membrane before the transition from a flat membrane to a highly curved invagination tip (Kukulski et al. 2012). This observation conflicts with the generally accepted dogma in which bud curvature is correlated with clathrin-coat growth (Ford et al. 2001, 2002).

\section{THE CELL-WIDE SPATIAL AND TEMPORAL DYNAMICS OF CCP NUCLEATION}

One of the great challenges of cell biology is to place molecular events with a spatial scale of nm and temporal scale $<1 \mathrm{sec}$ in the context of larger organelles with a spatial scale of $\mu \mathrm{m}$ and temporal scale $>1 \mathrm{sec}$. The cell-wide nucleation of endocytic buds at the plasma membrane is a case in point and an area of active debate.

The nucleation of mammalian CCPs was first monitored using live-cell fluorescence microscopy (either TIRF or Epi) combined with the expression of clathrin-FP or AP2-FP, which allowed the spatiotemporal dynamics of CCP nucleation to be measured (Gaidarov and Keen 1999). The moment of nucleation of a CCP was taken as the earliest detection of a fluorescent clathrin or AP2 spot, which subsequently grew in fluorescence as the CCP matured (Gaidarov and Keen 1999), although the actual moment of nucleation probably occurred 10-20 sec earlier (Ehrlich et al. 2004). In one set of results, mammalian CCP nucleation was found to be pseudorandom and it was proposed that the departure from complete spatial randomness was caused by an exclusion zone around nascent CCPs (Ehrlich et al. 2004).

In some cellular contexts, the site of repeated mammalian CME events was found to be clearly nonrandom and occurred preferentially at "hot spots" (Gaidarov et al. 1999; Merrifield et al. 2005; Cao et al. 2011; Nunez et al. 2011; Taylor et al. 2011). Indeed, the first study to describe the live-cell imaging of CCPs using Clc-GFP revealed multiple CCVs emanating from the same site without the host clathrin-coated structure (CCS) fully dimming (Gaidarov et al. 1999). These results were subsequently confirmed using a TIRF-based assay to detect single productive scission events, which showed that both punctate and larger plaque-like clathrin structures $(\sim 0.5-1 \mu \mathrm{m})$ in NIH-3T3 fibroblasts could host trains of multiple, quantized scission events (Merrifield et al. 2005; Taylor et al. 2011). Trains of scission events were spatially and temporally correlated with transient dimming of the host CCP, suggesting that only part of the coat participated in the budding event (Taylor et al. 2011). This would entail either remodeling of an existing flat clathrin lattice (den Otter and Briels 2011) or the addition of a curved domain to the flat lattice edge, as suggested by earlier EM images (Heuser 1980).

Even larger endocytic hotspots were revealed by live-cell confocal microscopy of Dyn2-GFP at the basal membrane of hepatocytes (Cao et al. 2011). In this instance, the endocytic hot spots occurred in $\sim 50 \%$ of cells with a lifetime of $\sim 10-30 \mathrm{~min}$, were $2-10 \mu \mathrm{m}$ in diameter, and could produce up to $10-15$ vesicles $/ \mathrm{min}$, in contrast to the $1-2$ vesicles/min supported by punctate CCPs. Ultrastructural analysis by EM revealed the hot spots comprised complex tubulovesicular networks with numerous budding clathrin coats. These structures are remarkably similar to the large, complex invaginations seen in neurons from dynamin knockout mice (Ferguson et al. 2007). It was suggested that the specialized endocytic plaques in hepatocytes may form transiently as clathrin and other endocytic factors concentrate above a threshold, although the trigger(s) responsible for the formation of such giant hot spots remain unclear.

It may be worth emphasizing here that the definition of "hot spot" is by no means fixed and apparently encompasses different, related phenomena. On the one hand, multiple CCVs can successively emerge in "trains" from fixed sites wherein the clathrin-FP signal never fully dims between budding events (Gaidarov et al. 1999; Merrifield et al. 2005; Cao et al. 2011; Taylor et al. 2011). On the other hand, the sequential nucleation of discrete CCPs can be both spatially and temporally correlated, suggesting that the plasma membrane has some form of "memory" for CCP nucleation (Nunez et al. 2011). Therefore that the term "hot spot" can mean both repeated endocytic events at the same CCS or repeated nucleation events of discrete CCPs at preferred sites at the plasma mem- 
brane. Precisely how these two different phenomena are related is currently unclear.

In yeast, the endocytic budding events are distributed in a nonrandom manner dependent on the cell-cycle state (Bi and Park 2012). Initially, in unbudded cells the endocytic sites are equally distributed throughout the cell, but when the budding starts the endocytic events become highly concentrated into the growing daughter cell. Later, during the cytokinesis, the endocytic sites concentrate to the cell division plane between the mother and daughter cells. This polarization of endocytic activity has an important role in the maintenance of the growth polarity (Jose et al. 2013).

At a smaller spatial scale the endocytic events in yeast appear random, except that they are excluded from plasma membrane regions that are in contact with cortical endoplasmic reticulum (Stradalova et al. 2012) and from eisosomes, stable microdomains of the fungal plasma membrane (Brach et al. 2011). The exclusion of endocytosis from these regions is most likely attributable to steric hindrance inhibiting bud nucleation.

\section{COAT MATURATION AND THE PROPAGATION OF MEMBRANE CURVATURE}

There is more than one mechanism to bend a membrane (Kirchhausen 2012; Shen et al. 2012) and therefore understanding precisely how
EAPs initiate, promote, and maintain membrane curvature at the forming endocytic bud in vivo remains a challenging problem. Generating curvature in a phospholipid bilayer implies that some form of packing asymmetry exists between the two leaflets of the membrane (Kirchhausen 2012). In principle, there are at least four ways in which cytosolic EAPs may contribute to lipid asymmetry and membrane curvature during CCP invagination: (1) by locally clustering lipids with larger-than-average head groups through interaction with membrane-associated proteins (Zimmerberg and Kozlov 2006), by converting one lipid type to another (Kooijman et al. 2003) or by actively transporting a lipid type(s) from one leaflet to the other (Rauch and Farge 2000; Roelants et al. 2010); (2) by "floating" an amphipathic helix in the inner leaflet of the membrane (Ford et al. 2002) or by inserting a hydrophobic loop into the inner leaflet of the membrane (Plomann et al. 2010); (3) by association of a precurved membrane-binding protein domain (such as BAR, F-BAR, or i-BAR) with the membrane surface (Mim et al. 2012); or, most simply, (4) by membrane-associated protein crowding (Stachowiak et al. 2012). The different mechanisms for generating membrane curvature are summarized in Figure 2.

Precisely which mechanism(s) plays a role in the different stages of CCP invagination and scission has been the subject of intense study and much of our understanding is based on in
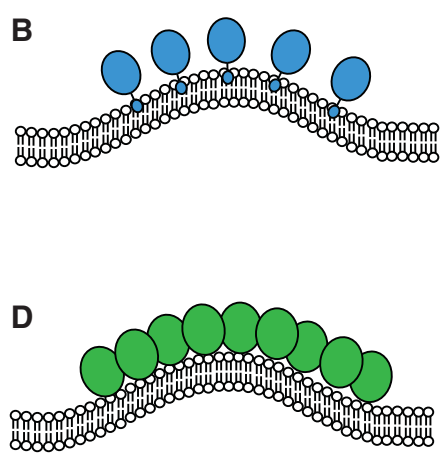

Figure 2. Different mechanisms of membrane bending. (A) Local clustering of lipids with large head groups. (B) Insertion of an amphipathic helix or a loop into the membrane. $(C)$ Binding of pre-curved proteins to the membrane surface. $(D)$ Crowding of membrane-associated proteins. 
vitro reconstitution assays using purified components (Dannhauser and Ungewickell 2012; Ford et al. 2002). A variety of different EAPs have been shown to induce membrane curvature and lipid tubulation (Fig. 2) (Shen et al. 2012) but, perhaps surprisingly, the minimal components for CCP budding in vitro are lipid, an (synthetic) adaptor, clathrin and dynamin (Dannhauser and Ungewickell 2012). The synthetic adaptor used in these classic experiments was a mutant of epsin wherein the membrane bending ENTH domain was replaced with a histidine tag $\left(\mathrm{H}_{6}-\Delta \mathrm{ENTH}-\mathrm{epsin}\right)$. Lipid vesicles, made from whole brain lipid, were doped with $\mathrm{Ni}^{2+}$-NTA-DOGS, which recruited $\mathrm{H}_{6}$ $\Delta \mathrm{ENTH}$-epsin to the liposome surface. In turn $\mathrm{H}_{6}-\Delta$ ENTH-epsin recruited clathrin, which, at $4^{\circ} \mathrm{C}$, formed flat lattices reminiscent of clathrin plaques found in mammalian cells (Heuser 1980; Saffarian et al. 2009; Dannhauser and Ungewickell 2012). However, and quite remarkably, on warming to $37^{\circ} \mathrm{C}$ the plaques reorganized into clathrin-coated buds of uniform size, which could be released from the liposome surface by dynamin (Dannhauser and Ungewickell 2012). At $37^{\circ} \mathrm{C}$, the plasma membrane is not a rigid sheet but undergoes low amplitude vibrations (Brown 2003) and although the characteristic length of membrane bending is short ( $50 \mathrm{~nm}$ ) (Brown 2003), it remains an intriguing idea that the clathrin lattice may act as a Brownian ratchet and rectify thermally induced flexing of the membrane (Hinrichsen et al. 2006). This is an attractive idea because, presumably, the clathrin lattice could effectively integrate a variety of curvature-inducing mechanisms giving rise to the vectorial nature of CCP formation (Hinrichsen et al. 2006).

The degree of invagination of isolated mammalian CCPs is tightly correlated with the amount of clathrin coating on the budding membrane, as shown by thin-section EM (Ehrlich et al. 2004). From this, one might predict the state of curvature of individual CCPs, labeled with Clc-FP, based on the relative fluorescence of clathrin spots (Ehrlich et al. 2004). However, in many cell types at optical resolution such measurements are complicated by the proximity of clathrin-coated buds to neighbor- ing clathrin-coated buds and/or flat clathrin lattices, at least at the basal surface of adherent cells (Heuser 1980; Hinrichsen et al. 2006). These are most likely endocytically active structures because, when individual scission events were detected in mammalian fibroblasts, it was shown that quantized scission events could be hosted by CCS of variable size and there was not a simple correlation between scission activity and the amplitude of Clc-FP signal (Merrifield et al. 2005; Taylor et al. 2011, 2012). At the time of writing, these findings have not been extended to explore the direct measurement of CCP curvature in real time and in live cells, although this is technically feasible (Sund et al. 1999).

\section{MEMBRANE SCISSION}

The final moment of endocytic vesicle formation is membrane scission wherein the thin membrane umbilicus linking an endocytic bud to the plasma membrane is severed (reviewed in Lenz et al. 2009; Campelo and Malhotra 2012). For mammalian cells, it is well established that membrane scission is controlled by the large GTPase dynamin and, because dynamin is perhaps one of the most extensively studied EAPs, rather than tackle the extensive literature, we will limit our discussion to a handful of recent key findings and refer the interested reader to several recent and extensive reviews (Mettlen et al. 2009; Ramachandran 2011; Schmid and Frolov 2011; Ferguson and De Camilli 2012; Morlot and Roux 2013; see also Johannes et al. 2014).

In the paradigm of dynamin action in mammalian cells, GTP-bound dynamin is recruited to the thin membrane neck of deeply invaginated CCPs where it executes membrane scission, most likely through GTP-hydrolysis-induced membrane compression (Morlot and Roux 2013). In a series of elegant biophysical studies using thin membrane tubules drawn from aspirated giant liposomes, Roux and colleagues showed that dynamin oligomerized on membrane tubules induced torque in the membrane tubule on the addition of GTP (Roux et al. 2006; Morlot and Roux 2013). It was shown that tubules sheared at the boundary between dynamin 
coated and uncoated sections of tubule (Morlot and Roux 2013). Membrane compression occurred rapidly following addition of GTP, as shown previously, but scission occurred much later with a longer delay following Poisson statistics. From these data, combined with careful modeling, the authors concluded that oligomerized dynamin compresses the membrane tubule to a diameter of $\sim 5 \mathrm{~nm}$ and developed stress at the boundary between the thin dynamin-coated section and thicker, uncoated adjacent regions. Critically, scission was found to be far more efficient if the membrane tubule was placed under tension, most likely because this eliminated the dissipation of dynamin-induced torque by membrane flow and instead favored membrane shearing (Morlot and Roux 2013). Most likely, a transient hemifused state leads to scission because the scission reaction is not leaky, as determined using elegant conductance measurements (Bashkirov et al. 2008). Overall, these results mesh well with recent studies publishing the crystal structure of dynamin, which provided detailed insight into the GTP-hydrolysis constriction mechanism of oligomerized dynamin (Chappie et al. 2011; Faelber et al. 2011, 2012; Ford et al. 2011).

The detailed analysis of the molecular mechanism of dynamin-induced membrane scission is a tour de force spread over several decades and involving many different labs (Mettlen et al. 2009; Ramachandran 2011; Schmid and Frolov 2011; Ferguson and De Camilli 2012; Morlot and Roux 2013). However, many questions remain concerning the mechanism of dynamin function and the roles of the numerous binding partners of dynamin including membrane curvature sensing/inducing BAR domain proteins such as amphiphysin and endophilin (Milosevic et al. 2011; Meinecke et al. 2013; Neumann and Schmid 2013) and proteins involved in actin polymerization/depolymerization (reviewed in Menon and Schafer 2013). Moreover, dynamin can also affect the earlier stages of CCP growth and invagination, although the precise mechanism by which it does so remains obscure (Loerke et al. 2009; Mettlen et al. 2009). Therefore the challenge remains to place the detailed molecular mechanism of dynamin membrane severing into the broader context of CCP maturation. One attractive idea is that dynamin works in concert with BAR domain proteins and the actin cytoskeleton to effect CCP invagination and that dynamin delivers the coup de grace to the resulting, strained membrane neck (Gu et al. 2010). This model is consistent with the discovery that in dynamin $1+2$ double knockout fibroblasts CCPs invaginate into the cytoplasm atop long membrane tubules coated with the BAR domain protein endophilin and actin, showing that invagination and actin polymerization still occur in the absence of dynamin but in a deregulated manner (Ferguson et al. 2009). This is congruent with a model in which dynamin is recruited after actin and endophilin have formed a thin membrane neck. More recently it was shown that dynamin GTPase mutants modulate actin dynamics at invaginating CCPs (Taylor et al. 2012) and, intriguingly, that dynamin itself can bind to actin and displace the capping protein gelsolin (Gu et al. 2010). These results suggest that there may be a yet more intimate relationship between dynamin and actin polymerization during CCP invagination and membrane scission. However, mammalian endocytosis does not always require actin polymerization.

Importantly, this model of the mammalian endocytic scission mechanism is consistent with a broad range of findings, but despite the compelling evidence that dynamin is necessary for the budding of CCPs some puzzling facts remain. It seems unlikely that dynamin-mediated scission represents a universal mechanism of vesicle budding because vesicles can bud from other membrane surfaces in the cell in a dynamin-independent manner (Campelo and Malhotra 2012). Moreover, in yeast, the dynamin homolog Vps1 is required for efficient endocytosis but is dispensable for endocytic vesicle budding per se, whereas the actin cytoskeleton is not (Aghamohammadzadeh and Ayscough 2009; Smaczynska-de et al. 2012). In mammalian cells the opposite is true and dynamin is essential for endocytosis, whereas the actin cytoskeleton is only essential in some cell types or under certain conditions (Aghamohammadzadeh and Ayscough 2009; Boulant et al. 2011). 
A second mechanism for effecting membrane scission is by the generation of a line tension in the membrane neck (Liu et al. 2006, 2009). Here, selective hydrolysis of PIP2 in the developing bud by the PI5' phosphatase synaptojanin [in mammalian cells (Chang-Ileto et al. 2011)] or Sjl2 [in yeast cells (Stefan et al. 2005)] leads to a line tension between PI4P enriched in the bud and PIP2 enriched in the tubular invagination, which remains protected by BAR domain proteins (in yeast) and dynamin (in mammalian cells) (Chang-Ileto et al. 2011). In yeast cells, this mechanism alone may be sufficient to effect scission of the deep, tubular invaginations formed, perhaps because a turgor pressure of $\sim 1 \mathrm{MPa}$ can help squeeze the nascent bud making a dynamin-like constriction mechanism redundant in the final stages of scission (Aghamohammadzadeh and Ayscough 2009). In mammalian cells, the same PIP2 hydrolysis mechanism most likely works in synergy with dynamin constriction and serves two roles: first to contribute to membrane instability by generating a line tension (Liu et al. 2006) and, second, to recruit the PI4P-binding G-cyclin-associated kinase (GAK), necessary for CCV uncoating, to the nascent bud (Lee et al. 2006). In this scenario, the coincidence of dynamin-constriction-mediated instability and a line tension would presumably work together to effect more efficient scission.

Overall, as for the mechanism(s) of membrane curvature, evolution has apparently blended these different mechanisms of membrane scission in different ways to suit the mechanochemical properties of the host cell. It will be of interest to explore this theme further in other types of walled cells, such as plant stromal guard cells, which have remarkably high turgor pressure of up to $\sim 4 \mathrm{MPa}$ (Meckel et al. 2005).

\section{UNCOATING}

Following scission, CCVs rapidly shed their clathrin coat through the coordinated action of the ATPase/chaperone Hsc70 (Chappell et al. 1986) and neuronal J-domain kinase (Ahle and Ungewickell 1990) or ubiquitous paralog auxilin2 (cyclin-G-associated kinase, GAK) (Greener et al. 2000; Umeda et al. 2000).

Live-cell imaging studies revealed GAK was recruited to sites of scission after dynamin (Lee et al. 2006; Massol et al. 2006; Taylor et al. 2011) and that GAK recruitment to budding CCPs required GAK's PI-binding PTEN domain (Lee et al. 2005; Massol et al. 2006). Full-length GAK was found to bind most tightly to monophosphorylated PIs, notably PI4P (Lee et al. 2005; Massol et al. 2006), which prompted the hypothesis that conversion of PIP2 to PI4P by a PI5' phosphatase might act as the trigger recruiting GAK to newly scissioned vesicles (Massol et al. 2006). Indeed it was already known that the $5^{\prime}$-PI phosphatase synaptojanin is required for CCV uncoating (Verstreken et al. 2003) and knockout in mice of auxilin $1+2$ (Yim et al. 2005; Lee et al. 2008) or synaptojanin (Cremona et al. 1999) similarly led to accumulation of synaptic CCVs. Loss of PIP2 from the newly scissioned vesicle via synaptojanin-mediated PIP2 dephosphorylation would explain why CCVs also lose their AP2 shell because binding of AP2 to the membrane is mediated by PIP2 (Honing et al. 2005). This led to a model in which the coordinated action of synaptojanin (to generate PI4P and abolish AP-membrane interaction) and auxilin/GAK/Hsc70 (to remove clathrin) act together to irreversibly shed the clathrin/adaptor coat of newly formed CCVs (Massol et al. 2006).

One feature of this model that remained unclear was how auxilin / GAK recruitment and synaptojanin recruitment were coordinated. It was previously established that the BAR domain protein endophilin targets synaptojanin to newly scissioned vesicles in Drosophila (Verstreken et al. 2002). This was later confirmed by the generation of endophilin knockout mice by the de Camilli lab (Milosevic et al. 2011). Here, knockout of all three endophilin isoforms led to the accumulation of CCVs, but not CCPs, in mouse synapses (Milosevic et al. 2011).

\section{CONCLUSIONS AND FUTURE DIRECTIONS}

One of the most fascinating properties of the endocytic machinery is that, quite unlike any 
man-made machine, the machinery self-assembles in a stereotyped manner in the absence of any one "manager" and despite stochastic variation inherent in the small numbers of molecules involved (Sirotkin et al. 2010). Also, the endocytic machinery is remarkably robust in the sense that particular components can be ablated or mutated and the machinery will often still work, albeit with altered kinetics (Kaksonen et al. 2005). Understanding the design principles underlying the function of this prototypical self-assembling, a robust molecular machine remains a major challenge in the field.

Most of the experiments described in this review were designed to investigate the properties and mechanistic action of one or a few specific components of the endocytic machinery, often in isolation, and these types of experiments have been essential in understanding how the endocytic machinery works. However, the field has reached a stage of maturity in which diverse studies on different EAPs can be integrated into concise, predictive models. In an elegant paper, Drubin (an experimentalist) and Oster (a theorist) and colleagues integrated ultrastructural, biochemical, and live-cell imaging data of the endocytic machinery in yeast into a mechanochemical model of endocytosis (Liu et al. 2009). The model helps explain how, at each moment of the invagination and scission process, feedback loops between changes in membrane geometry, membrane composition, and EAP recruitment seamlessly set the scene for the next moment in the process. Perhaps the future of studies in endocytosis, and membrane trafficking in general, is to develop and rigorously test these types of models and ask how subtle changes in endocytic membrane trafficking may contribute to the development of different disease pathologies over the lifetime of an individual.

Building such predictive models will require systematic, quantitative data on the endocytic machinery including both reductionist data about individual components obtained from in vitro systems and more holistic data from intact cells. Combining these types of approaches will likely be essential for a comprehensive mechanistic model of the endocytic process.

\section{REFERENCES}

* Reference is also in this collection.

Abe N, Inoue T, Galvez T, Klein L, Meyer T. 2008. Dissecting the role of PtdIns $(4,5) \mathrm{P}_{2}$ in endocytosis and recycling of the transferrin receptor. J Cell Sci 121: 1488-1494.

Aghamohammadzadeh S, Ayscough KR. 2009. Differential requirements for actin during yeast and mammalian endocytosis. Nat Cell Biol 11: 1039-1042.

Aguet F, Antonescu CN, Mettlen M, Schmid SL, Danuser G. 2013. Advances in analysis of low signal-to-noise images link dynamin and AP2 to the functions of an endocytic checkpoint. Dev Cell 26: 279-291.

Ahle S, Ungewickell E. 1990. Auxilin, a newly identified clathrin-associated protein in coated vesicles from bovine brain. J Cell Biol 111: 19-29.

Antonescu CN, Danuser G, Schmid SL. 2010. Phosphatidic acid plays a regulatory role in clathrin-mediated endocytosis. Mol Biol Cell 21: 2944-2952.

Bashkirov PV, Akimov SA, Evseev AI, Schmid SL, Zimmerberg J, Frolov VA. 2008. GTPase cycle of dynamin is coupled to membrane squeeze and release, leading to spontaneous fission. Cell 135: 1276-1286.

Bi E, Park HO. 2012. Cell polarization and cytokinesis in budding yeast. Genetics 191: 347-387.

* Bökel C, Brand M. 2014. Endocytosis and signaling during development. Cold Spring Harb Perspect Biol doi: 10.1101/cshperspect.a017020.

Boucrot E, Saffarian S, Zhang R, Kirchhausen T. 2010. Roles of AP2 in clathrin-mediated endocytosis. PloS ONE 5: e10597.

Boulant S, Kural C, Zeeh JC, Ubelmann F, Kirchhausen T. 2011. Actin dynamics counteract membrane tension during clathrin-mediated endocytosis. Nat Cell Biol 13: 1124-1131.

Brach T, Specht T, Kaksonen M. 2011. Reassessment of the role of plasma membrane domains in the regulation of vesicular traffic in yeast. J Cell Sci 124: 328-337.

Brown FL. 2003. Regulation of protein mobility via thermal membrane undulations. Biophys J 84: 842-853.

Burston HE, Maldonado-Baez L, Davey M, Montpetit B, Schluter C, Wendland B, Conibear E. 2009. Regulators of yeast endocytosis identified by systematic quantitative analysis. J Cell Biol 185: 1097-1110.

Campelo F, Malhotra V. 2012. Membrane fission: The biogenesis of transport carriers. Ann Rev Biochem 81: 407-427.

Cao H, Krueger EW, McNiven MA. 2011. Hepatocytes internalize trophic receptors at large endocytic "hot spots." Hepatology 54: 1819-1829.

Carroll SY, Stirling PC, Stimpson HE, Giesselmann E, Schmitt MJ, Drubin DG. 2009. A yeast killer toxin screen provides insights into a/b toxin entry, trafficking, and killing mechanisms. Dev Cell 17: 552-560.

Carroll SY, Stimpson HE, Weinberg J, Toret CP, Sun Y, Drubin DG. 2012. Analysis of yeast endocytic site formation and maturation through a regulatory transition point. Mol Biol Cell 23: 657-668.

Chang-Ileto B, Frere SG, Chan RB, Voronov SV, Roux A, Di Paolo G. 2011. Synaptojanin 1-mediated PI(4,5) $\mathrm{P}_{2}$ hy- 
drolysis is modulated by membrane curvature and facilitates membrane fission. Dev Cell 20: 206-218.

Chappell TG, Welch WJ, Schlossman DM, Palter KB, Schlesinger MJ, Rothman JE. 1986. Uncoating ATPase is a member of the 70 kilodalton family of stress proteins. Cell 45: 3-13.

Chappie JS, Mears JA, Fang S, Leonard M, Schmid SL, Milligan RA, Hinshaw JE, Dyda F. 2011. A pseudoatomic model of the dynamin polymer identifies a hydrolysisdependent powerstroke. Cell 147: 209-222.

Chen X, Irani NG, Friml J. 2011. Clathrin-mediated endocytosis: The gateway into plant cells. Curr Opin Plant Biol 14: $674-682$.

Cocucci E, Aguet F, Boulant S, Kirchhausen T. 2012. The first five seconds in the life of a clathrin-coated pit. Cell 150: 495-507.

* Cosker KE, Segal RA. 2014. Neuronal signaling through endocytosis. Cold Spring Harb Perspect Biol 6: a020669.

Cremona O, Di Paolo G, Wenk MR, Luthi A, Kim WT, Takei K, Daniell L, Nemoto Y, Shears SB, Flavell RA, et al. 1999. Essential role of phosphoinositide metabolism in synaptic vesicle recycling. Cell 99: 179-188.

Dannhauser PN, Ungewickell EJ. 2012. Reconstitution of clathrin-coated bud and vesicle formation with minimal components. Nat Cell Biol 14: 634-639.

den Otter WK, Briels WJ. 2011. The generation of curved clathrin coats from flat plaques. Traffic 12: 1407-1416.

* Di Fiore PP, von Zastrow M. 2014. Endocytosis, signaling, and beyond. Cold Spring Harb Perspect Biol doi: 10.1101/ cshperspect.a016865.

Doyon JB, Zeitler B, Cheng J, Cheng AT, Cherone JM, Santiago Y, Lee AH, Vo TD, Doyon Y, Miller JC, et al. 2011. Rapid and efficient clathrin-mediated endocytosis revealed in genome-edited mammalian cells. Nat Cell Biol 13: $331-337$.

Ehrlich M, Boll W, Van Oijen A, Hariharan R, Chandran K, Nibert ML, Kirchhausen T. 2004. Endocytosis by random initiation and stabilization of clathrin-coated pits. Cell 118: 591-605.

Faelber K, Posor Y, Gao S, Held M, Roske Y, Schulze D, Haucke V, Noe F, Daumke O. 2011. Crystal structure of nucleotide-free dynamin. Nature 477: 556-560.

Faelber K, Held M, Gao S, Posor Y, Haucke V, Noe F, Daumke O. 2012. Structural insights into dynamin-mediated membrane fission. Structure 20: 1621-1628.

Ferguson SM, De Camilli P. 2012. Dynamin, a membraneremodelling GTPase. Nat Rev Mol Cell Biol 13: 75-88.

Ferguson SM, Brasnjo G, Hayashi M, Wolfel M, Collesi C, Giovedi S, Raimondi A, Gong LW, Ariel P, Paradise S, 2007. A selective activity-dependent requirement for dynamin 1 in synaptic vesicle endocytosis. Science 316: $570-574$.

Ferguson SM, Raimondi A, Paradise S, Shen H, Mesaki K, Ferguson A, Destaing O, Ko G, Takasaki J, Cremona O, et al. 2009. Coordinated actions of actin and BAR proteins upstream of dynamin at endocytic clathrin-coated pits. Dev Cell 17: 811-822.

Ford MG, Pearse BM, Higgins MK, Vallis Y, Owen DJ, Gibson A, Hopkins CR, Evans PR, McMahon HT. 2001. Simultaneous binding of $\operatorname{PtdIns}(4,5) \mathrm{P}_{2}$ and clathrin by
AP180 in the nucleation of clathrin lattices on membranes. Science 291: 1051-1055.

Ford MG, Mills IG, Peter BJ, Vallis Y, Praefcke GJ, Evans PR, McMahon HT. 2002. Curvature of clathrin-coated pits driven by epsin. Nature 419: 361-366.

Ford MG, Jenni S, Nunnari J. 2011. The crystal structure of dynamin. Nature 477: 561-566.

Fujita A, Cheng J, Tauchi-Sato K, Takenawa T, Fujimoto T. 2009. A distinct pool of phosphatidylinositol 4,5-bisphosphate in caveolae revealed by a nanoscale labeling technique. Proc Natl Acad Sci 106: 9256-9261.

Gaidarov I, Keen JH. 1999. Phosphoinositide-AP-2 interactions required for targeting to plasma membrane clathrin-coated pits. J Cell Biol 146: 755-764.

Gaidarov I, Santini F, Warren RA, Keen JH. 1999. Spatial control of coated-pit dynamics in living cells. Nat Cell Biol 1: 1-7.

Goh LK, Huang F, Kim W, Gygi S, Sorkin A. 2010. Multiple mechanisms collectively regulate clathrin-mediated endocytosis of the epidermal growth factor receptor. J Cell Biol 189: 871-883.

Greener T, Zhao X, Nojima H, Eisenberg E, Greene LE. 2000. Role of cyclin G-associated kinase in uncoating clathrincoated vesicles from non-neuronal cells. J Biol Chem 275: $1365-1370$.

Gu C, Yaddanapudi S, Weins A, Osborn T, Reiser J, Pollak M, Hartwig J, Sever S. 2010. Direct dynamin-actin interactions regulate the actin cytoskeleton. EMBO J 29: $3593-$ 3606.

Hammond GR, Fischer MJ, Anderson KE, Holdich J, Koteci A, Balla T, Irvine RF. 2012. PI4P and PI $(4,5) \mathrm{P}_{2}$ are essential but independent lipid determinants of membrane identity. Science 337: 727-730.

Henne WM, Kent HM, Ford MG, Hegde BG, Daumke O, Butler PJ, Mittal R, Langen R, Evans PR, McMahon HT. 2007. Structure and analysis of FCHo2 F-BAR domain: A dimerizing and membrane recruitment module that effects membrane curvature. Structure 15: 839-852.

Henne WM, Boucrot E, Meinecke M, Evergren E, Vallis Y, Mittal R, McMahon HT. 2010. FCHo proteins are nucleators of clathrin-mediated endocytosis. Science 328: 1281-1284.

Heuser J. 1980. Three-dimensional visualization of coated vesicle formation in fibroblasts. J Cell Biol 84: 560-583.

Hinrichsen L, Meyerholz A, Groos S, Ungewickell EJ. 2006. Bending a membrane: How clathrin affects budding. Proc Natl Acad Sci 103: 8715-8720.

Honing S, Ricotta D, Krauss M, Spate K, Spolaore B, Motley A, Robinson M, Robinson C, Haucke V, Owen DJ. 2005. Phosphatidylinositol-(4,5)-bisphosphate regulates sorting signal recognition by the clathrin-associated adaptor complex AP2. Mol Cell 18: 519-531.

Huang F, Khvorova A, Marshall W, Sorkin A. 2004. Analysis of clathrin-mediated endocytosis of epidermal growth factor receptor by RNA interference. J Biol Chem 279: 16657-16661.

Idrissi FZ, Blasco A, Espinal A, Geli MI. 2012. Ultrastructural dynamics of proteins involved in endocytic budding. Proc Natl Acad Sci 109: E2587-E2594.

Jackson LP, Kelly BT, McCoy AJ, Gaffry T, James LC, Collins BM, Honing S, Evans PR, Owen DJ. 2010. A large-scale 
conformational change couples membrane recruitment to cargo binding in the AP2 clathrin adaptor complex. Cell 141: 1220-1229.

* Johannes L, Wunder C, Bassereau P. 2014. Bending "on the rocks"-A cocktail of biophysical modules to build endocytic pathways. Cold Spring Harb Perspect Biol 6: a016741.

Jose M, Tollis S, Nair D, Sibarita JB, McCusker D. 2013. Robust polarity establishment occurs via an endocytosis-based cortical corralling mechanism. J Cell Biol 200: 407-418.

Jost M, Simpson F, Kavran JM, Lemmon MA, Schmid SL 1998. Phosphatidylinositol-4,5-bisphosphate is required for endocytic coated vesicle formation. Curr Biol 8: 1399_ 1402.

Kaksonen M, Toret CP, Drubin DG. 2005. A modular design for the clathrin- and actin-mediated endocytosis machinery. Cell 123: 305-320.

Kirchhausen T. 2009. Imaging endocytic clathrin structures in living cells. Trend Cell Biol 19: 596-605.

Kirchhausen T. 2012. Bending membranes. Nat Cell Biol 14: 906-908.

Kishimoto T, Sun Y, Buser C, Liu J, Michelot A, Drubin DG 2011. Determinants of endocytic membrane geometry, stability, and scission. Proc Natl Acad Sci 108: E979E988.

Kooijman EE, Chupin V, de Kruijff B, Burger KN. 2003. Modulation of membrane curvature by phosphatidic acid and lysophosphatidic acid. Traffic 4: 162-174.

Kukulski W, Schorb M, Kaksonen M, Briggs JA. 2012. Plasma membrane reshaping during endocytosis is revealed by time-resolved electron tomography. Cell 150: $508-$ 520.

Lee DW, Zhao X, Zhang F, Eisenberg E, Greene LE. 2005. Depletion of GAK/auxilin 2 inhibits receptor-mediated endocytosis and recruitment of both clathrin and clathrin adaptors. J Cell Sci 118: 4311-4321.

Lee DW, Wu X, Eisenberg E, Greene LE. 2006. Recruitment dynamics of GAK and auxilin to clathrin-coated pits during endocytosis. J Cell Sci 119: 3502-3512.

Lee DW, Zhao X, Yim YI, Eisenberg E, Greene LE. 2008. Essential role of cyclin-G-associated kinase (auxilin-2) in developing and mature mice. Mol Biol Cell 19: 2766-2776.

Lenz M, Morlot S, Roux A. 2009. Mechanical requirements for membrane fission: Common facts from various examples. FEBS Lett 583: 3839-3846.

Liu J, Kaksonen M, Drubin DG, Oster G. 2006. Endocytic vesicle scission by lipid phase boundary forces. Proc Natl Acad Sci 103: 10277-10282.

Liu J, Sun Y, Drubin DG, Oster GF. 2009. The mechanochemistry of endocytosis. PLoS Biol 7: e1000204.

Liu AP, Aguet F, Danuser G, Schmid SL. 2010. Local clustering of transferrin receptors promotes clathrin-coated pit initiation. J Cell Biol 191: 1381-1393.

Loerke D, Mettlen M, Yarar D, Jaqaman K, Jaqaman H, Danuser G, Schmid SL. 2009. Cargo and dynamin regulate clathrin-coated pit maturation. PLoS Biol 7: e57.

Mao Y, Balkin DM, Zoncu R, Erdmann KS, Tomasini L, Hu F, Jin MM, Hodsdon ME, De Camilli P. 2009. A $\mathrm{PH}$ domain within OCRL bridges clathrin-mediated membrane trafficking to phosphoinositide metabolism. EMBO J 28: 1831-1842.

Massol RH, Boll W, Griffin AM, Kirchhausen T. 2006. A burst of auxilin recruitment determines the onset of clathrin-coated vesicle uncoating. Proc Natl Acad Sci 103: 10265-10270.

McMahon HT, Boucrot E. 2011. Molecular mechanism and physiological functions of clathrin-mediated endocytosis. Nat Rev Mol Cell Biol 12: 517-533.

Meckel T, Hurst AC, Thiel G, Homann U. 2004. Endocytosis against high turgor: Intact guard cells of Vicia faba constitutively endocytose fluorescently labelled plasma membrane and GFP-tagged K-channel KAT1. Plant J 39: $182-193$.

Meckel T, Hurst AC, Thiel G, Homann U. 2005. Guard cells undergo constitutive and pressure-driven membrane turnover. Protoplasma 226: 23-29.

Meinecke M, Boucrot E, Camdere G, Hon WC, Mittal R, McMahon HT. 2013. Cooperative recruitment of dynamin and BIN/amphiphysin/Rvs (BAR) domain-containing proteins leads to GTP-dependent membrane scission. J Biol Chem 288: 6651-6661.

Menon M, Schafer DA. 2013. Dynamin: Expanding its scope to the cytoskeleton. Int Rev Cell Mol Biol 302: 187-219.

Merrifield CJ. 2012. Fishing for clathrin-coated pit nucleators. Nat Cell Biol 14: 452-454.

Merrifield CJ, Perrais D, Zenisek D. 2005. Coupling between clathrin-coated-pit invagination, cortactin recruitment, and membrane scission observed in live cells. Cell 121: 593-606.

Mettlen M, Pucadyil T, Ramachandran R, Schmid SL. 2009. Dissecting dynamin's role in clathrin-mediated endocytosis. Biochem Soc Trans 37: 1022-1026.

Milosevic I, Giovedi S, Lou X, Raimondi A, Collesi C, Shen H, Paradise S, O'Toole E, Ferguson S, Cremona O, et al. 2011. Recruitment of endophilin to clathrin-coated pit necks is required for efficient vesicle uncoating after fission. Neuron 72: 587-601.

Mim C, Cui H, Gawronski-Salerno JA, Frost A, Lyman E, Voth GA, Unger VM. 2012. Structural basis of membrane bending by the N-BAR protein endophilin. Cell 149: 137-145.

Morlot S, Roux A. 2013. Mechanics of dynamin-mediated membrane fission. Ann Rev Biophys 42: 629-649.

Motley A, Bright NA, Seaman MN, Robinson MS. 2003. Clathrin-mediated endocytosis in AP2-depleted cells. J Cell Biol 162: 909-918.

Nakatsu F, Perera RM, Lucast L, Zoncu R, Domin J, Gertler FB, Toomre D, De Camilli P. 2010. The inositol 5-phosphatase SHIP2 regulates endocytic clathrin-coated pit dynamics. J Cell Biol 190: 307-315.

Nakatsu F, Baskin JM, Chung J, Tanner LB, Shui G, Lee SY, Pirruccello M, Hao M, Ingolia NT, Wenk MR, et al. 2012. PtdIns4P synthesis by PI4KIII $\alpha$ at the plasma membrane and its impact on plasma membrane identity. J Cell Biol 199: 1003-1016.

Neumann S, Schmid SL. 2013. Dual role of BAR domaincontaining proteins in regulating dynamin-2 catalyzed vesicle release. J Biol Chem 288: 25119-25128.

Nunez D, Antonescu C, Mettlen M, Liu A, Schmid SL, Loerke D, Danuser G. 2011. Hotspots organize clathrin- 
mediated endocytosis by efficient recruitment and retention of nucleating resources. Traffic 12: 1868-1878.

Payne GS, Baker D, van Tuinen E, Schekman R. 1988. Protein transport to the vacuole and receptor-mediated endocytosis by clathrin heavy chain-deficient yeast. J Cell Biol 106: $1453-1461$.

Perera RM, Zoncu R, Lucast L, De Camilli P, Toomre D. 2006. Two synaptojanin 1 isoforms are recruited to clathrin-coated pits at different stages. Proc Natl Acad Sci 103: 19332-19337.

Plomann M, Wittmann JG, Rudolph MG. 2010. A hinge in the distal end of the PACSIN 2 F-BAR domain may contribute to membrane-curvature sensing. J Mol Biol 400: $129-136$.

Posor Y, Eichhorn-Gruenig M, Puchkov D, Schoneberg J, Ullrich A, Lampe A, Muller R, Zarbakhsh S, Gulluni F, Hirsch E, et al. 2013. Spatiotemporal control of endocytosis by phosphatidylinositol-3,4-bisphosphate. Nature 499: $233-237$.

Ramachandran R. 2011. Vesicle scission: Dynamin. Sem Cell Dev Biol 22: 10-17.

Rauch C, Farge E. 2000. Endocytosis switch controlled by transmembrane osmotic pressure and phospholipid number asymmetry. Biophys J 78: 3036-3047.

Reider A, Barker SL, Mishra SK, Im YJ, Maldonado-Baez L, Hurley JH, Traub LM, Wendland B. 2009. Syp1 is a conserved endocytic adaptor that contains domains involved in cargo selection and membrane tubulation. EMBO J 28: 3103-3116.

Roelants FM, Baltz AG, Trott AE, Fereres S, Thorner J. 2010. A protein kinase network regulates the function of aminophospholipid flippases. Proc Natl Acad Sci 107: 34-39.

Roux A, Uyhazi K, Frost A, De Camilli P. 2006. GTP-dependent twisting of dynamin implicates constriction and tension in membrane fission. Nature 441: 528-531.

Saffarian S, Cocucci E, Kirchhausen T. 2009. Distinct dynamics of endocytic clathrin-coated pits and coated plaques. PLoS Biol 7: e1000191.

Sakaushi S, Inoue K, Zushi H, Senda-Murata K, Fukada T, Oka S, Sugimoto K. 2007. Dynamic behavior of FCHO1 revealed by live-cell imaging microscopy: Its possible involvement in clathrin-coated vesicle formation. Biosci Biotechnol Biochem 71: 1764-1768.

Schmid SL, Frolov VA. 2011. Dynamin: Functional design of a membrane fission catalyst. Ann Rev Cell Dev Biol 27: 79-105.

Shen H, Pirruccello M, De Camilli P. 2012. SnapShot: Membrane curvature sensors and generators. Cell 150: e13011302.

Shimada A, Niwa H, Tsujita K, Suetsugu S, Nitta K, Hanawa-Suetsugu K, Akasaka R, Nishino Y, Toyama M, Chen L, et al. 2007. Curved EFC/F-BAR-domain dimers are joined end to end into a filament for membrane invagination in endocytosis. Cell 129: 761-772.

Sigismund S, Confalonieri S, Ciliberto A, Polo S, Scita G, Di Fiore PP. 2012. Endocytosis and signaling: Cell logistics shape the eukaryotic cell plan. Physiol Rev 92: 273-366.

Sirotkin V, Berro J, Macmillan K, Zhao L, Pollard TD. 2010. Quantitative analysis of the mechanism of endocytic actin patch assembly and disassembly in fission yeast. Mol Biol Cell 21: 2894-2904.
Smaczynska-de R II, Allwood EG, Mishra R, Booth WI, Aghamohammadzadeh S, Goldberg MW, Ayscough KR. 2012. Yeast dynamin Vps1 and amphiphysin Rvs 167 function together during endocytosis. Traffic 13: 317-328.

Stachowiak JC, Schmid EM, Ryan CJ, Ann HS, Sasaki DY, Sherman MB, Geissler PL, Fletcher DA, Hayden CC. 2012. Membrane bending by protein-protein crowding. Nat Cell Biol 14: 944-949.

Stefan CJ, Padilla SM, Audhya A, Emr SD. 2005. The phosphoinositide phosphatase Sjl2 is recruited to cortical actin patches in the control of vesicle formation and fission during endocytosis. Mol Cell Biol 25: 2910-2923.

Stradalova V, Blazikova M, Grossmann G, Opekarova M, Tanner W, Malinsky J. 2012. Distribution of cortical endoplasmic reticulum determines positioning of endocytic events in yeast plasma membrane. PloS ONE 7: e35132.

Sun Y, Drubin DG. 2012. The functions of anionic phospholipids during clathrin-mediated endocytosis site initiation and vesicle formation. J Cell Sci 125: 6157-6165.

Sun Y, Carroll S, Kaksonen M, Toshima JY, Drubin DG. 2007. PtdIns $(4,5) \mathrm{P}_{2}$ turnover is required for multiple stages during clathrin- and actin-dependent endocytic internalization. J Cell Biol 177: 355-367.

Sund SE, Swanson JA, Axelrod D. 1999. Cell membrane orientation visualized by polarized total internal reflection fluorescence. Biophys J 77: 2266-2283.

Tan PK, Davis NG, Sprague GF, Payne GS. 1993. Clathrin facilitates the internalization of seven transmembrane segment receptors for mating pheromones in yeast. $J$ Cell Biol 123: 1707-1716.

Taylor MJ, Perrais D, Merrifield CJ. 2011. A high precision survey of the molecular dynamics of mammalian clathrin-mediated endocytosis. PLoS Biol 9: e1000604.

Taylor MJ, Lampe M, Merrifield CJ. 2012. A feedback loop between dynamin and actin recruitment during clathrinmediated endocytosis. PLoS Biol 10: e1001302.

Toshima JY, Toshima J, Kaksonen M, Martin AC, King DS, Drubin DG. 2006. Spatial dynamics of receptor-mediated endocytic trafficking in budding yeast revealed by using fluorescent $\alpha$-factor derivatives. Proc Natl Acad Sci 103: 5793-5798.

Umasankar PK, Sanker S, Thieman JR, Chakraborty S, Wendland B, Tsang M, Traub LM. 2012. Distinct and separable activities of the endocytic clathrin-coat components Fchol $/ 2$ and AP- 2 in developmental patterning. Nat Cell Biol 14: 488-501.

Umeda A, Meyerholz A, Ungewickell E. 2000. Identification of the universal cofactor (auxilin 2) in clathrin coat dissociation. Eur J Cell Biol 79: 336-342.

Verstreken P, Kjaerulff O, Lloyd TE, Atkinson R, Zhou Y, Meinertzhagen IA, Bellen HJ. 2002. Endophilin mutations block clathrin-mediated endocytosis but not neurotransmitter release. Cell 109: 101-112.

Verstreken P, Koh TW, Schulze KL, Zhai RG, Hiesinger PR, Zhou Y, Mehta SQ, Cao Y, Roos J, Bellen HJ. 2003. Synaptojanin is recruited by endophilin to promote synaptic vesicle uncoating. Neuron 40: 733-48.

Weinberg J, Drubin DG. 2012. Clathrin-mediated endocytosis in budding yeast. Trend Cell Biol 22: 1-13. 


\section{C.J. Merrifield and M. Kaksonen}

Wettey FR, Jackson AP. 2006. Using DT40 to study clathrin function. Subcell Biochem 40: 119-143.

Yim YI, Scarselletta S, Zang F, Wu X, Lee DW, Kang YS, Eisenberg E, Greene LE. 2005. Exchange of clathrin, AP2 and epsin on clathrin-coated pits in permeabilized tissue culture cells. J Cell Sci 118: 2405-2413.
Zimmerberg J, Kozlov MM. 2006. How proteins produce cellular membrane curvature. Nat Rev Mol Cell Biol 7: 9-19.

Zoncu R, Perera RM, Balkin DM, Pirruccello M, Toomre D, De Camilli P. 2009. A phosphoinositide switch controls the maturation and signaling properties of APPL endosomes. Cell 136: 1110-1121. 


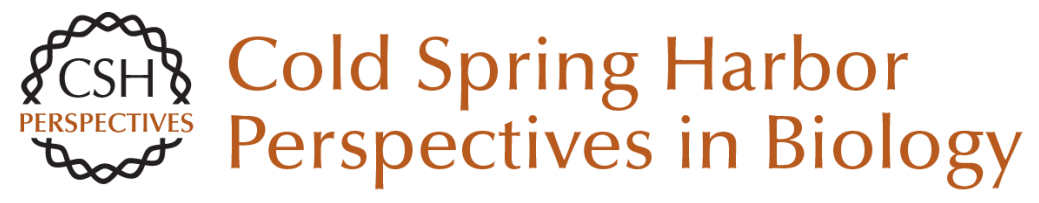

\section{Endocytic Accessory Factors and Regulation of Clathrin-Mediated Endocytosis}

Christien J. Merrifield and Marko Kaksonen

Cold Spring Harb Perspect Biol 2014; doi: 10.1101/cshperspect.a016733 originally published online October 3, 2014

\section{Subject Collection Endocytosis}

Endocytosis: Past, Present, and Future Sandra L. Schmid, Alexander Sorkin and Marino Zerial

Rab Proteins and the Compartmentalization of the Endosomal System Angela Wandinger-Ness and Marino Zerial

Cargo Sorting in the Endocytic Pathway: A Key Regulator of Cell Polarity and Tissue Dynamics Suzanne Eaton and Fernando Martin-Belmonte

Unconventional Functions for Clathrin, ESCRTs, and Other Endocytic Regulators in the

Cytoskeleton, Cell Cycle, Nucleus, and Beyond:

Links to Human Disease

Frances M. Brodsky, R. Thomas Sosa, Joel A. Ybe, et al.

Endocytosis of Viruses and Bacteria Pascale Cossart and Ari Helenius

Lysosomal Adaptation: How the Lysosome

Responds to External Cues

Carmine Settembre and Andrea Ballabio

Reciprocal Regulation of Endocytosis and Metabolism

Costin N. Antonescu, Timothy E. McGraw and Amira Klip
Imaging and Modeling the Dynamics of

Clathrin-Mediated Endocytosis Marcel Mettlen and Gaudenz Danuser

Endocytic Accessory Factors and Regulation of Clathrin-Mediated Endocytosis

Christien J. Merrifield and Marko Kaksonen

The Complex Ultrastructure of the Endolysosomal System

Judith Klumperman and Graça Raposo

The Biogenesis of Lysosomes and

Lysosome-Related Organelles

J. Paul Luzio, Yvonne Hackmann, Nele M.G.

Dieckmann, et al.

Endocytosis, Signaling, and Beyond Pier Paolo Di Fiore and Mark von Zastrow

Clathrin-Independent Pathways of Endocytosis Satyajit Mayor, Robert G. Parton and Julie G. Donaldson

The Role of Endocytosis during Morphogenetic Signaling Marcos Gonzalez-Gaitan and Frank Jülicher

For additional articles in this collection, see http://cshperspectives.cshlp.org/cgi/collection/

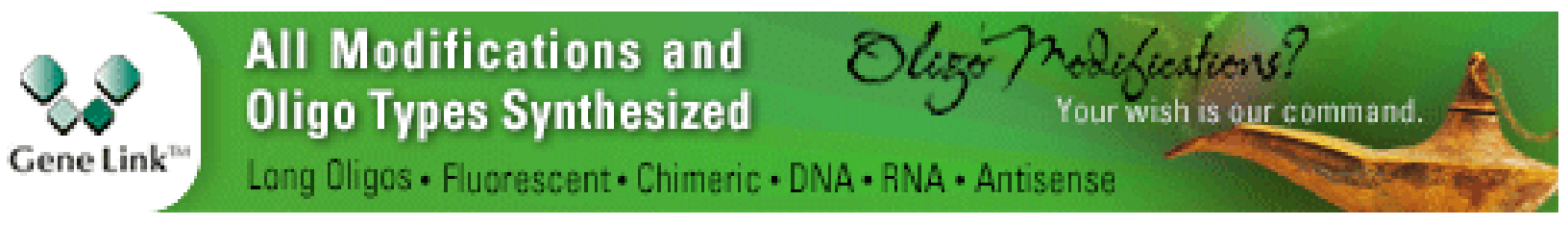




\section{Endocytosis and Autophagy: Exploitation or Cooperation?}

Sharon A. Tooze, Adi Abada and Zvulun Elazar
Role of Endosomes and Lysosomes in Human Disease

Frederick R. Maxfield

For additional articles in this collection, see http://cshperspectives.cshlp.org/cgi/collection/

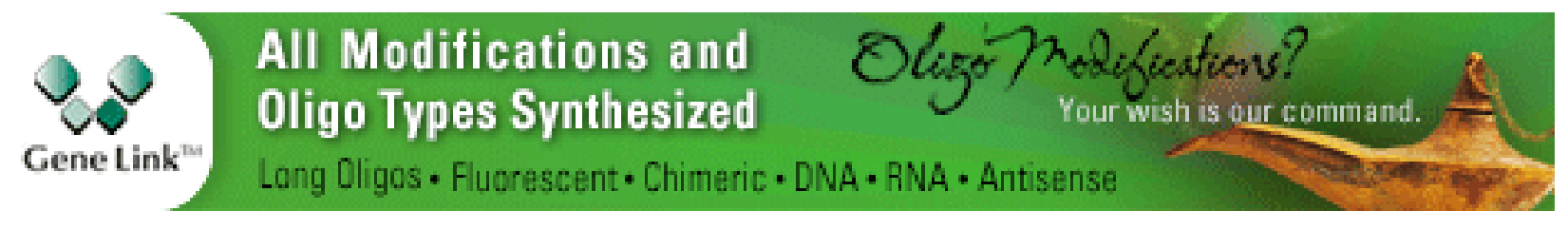

Copyright @ 2014 Cold Spring Harbor Laboratory Press; all rights reserved 